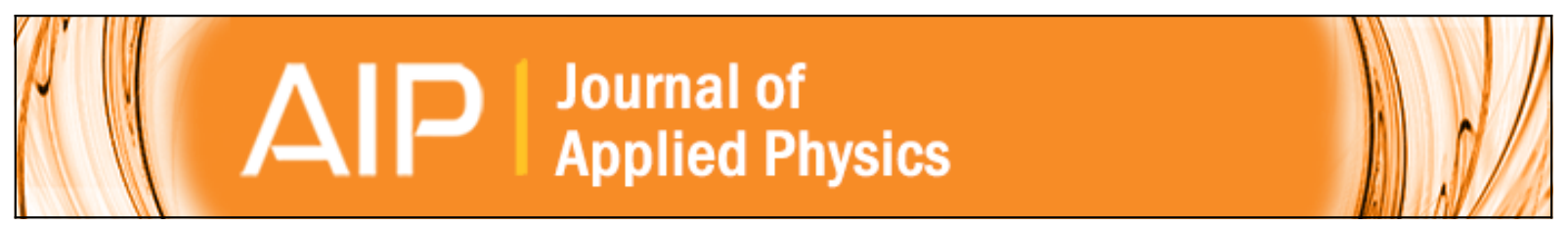

\title{
Ferromagnetic and multiferroic interfaces in granular perovskite composite
}

\section{xLa0.5Sr0.5CoO3-(1-x)BiFeO3}

Javier H. Lohr, Carlos A. López, Martín E. Saleta, and Rodolfo D. Sánchez

Citation: Journal of Applied Physics 120, 074103 (2016); doi: 10.1063/1.4960697

View online: http://dx.doi.org/10.1063/1.4960697

View Table of Contents: http://scitation.aip.org/content/aip/journal/jap/120/7?ver=pdfcov

Published by the AIP Publishing

\section{Articles you may be interested in}

Multiferroic and magnetoelectric properties of $\mathrm{CoFe} 2 \mathrm{O} 4 / \mathrm{Pb} 1-x \mathrm{SrxTiO} 3$ composite films

J. Appl. Phys. 117, 164101 (2015); 10.1063/1.4918663

Multiferroic Ni0.6Zn0.4Fe2O4-BaTiO3 nanostructures: Magnetoelectric coupling, dielectric, and fluorescence J. Appl. Phys. 116, 124103 (2014); 10.1063/1.4896118

Room-temperature magnetoelectric coupling in nanocrystalline $\mathrm{Na} 0.5 \mathrm{Bi0} 0.5 \mathrm{TiO} 3$

J. Appl. Phys. 116, 083909 (2014); 10.1063/1.4893720

Magnetoelectric coupling in solution derived 3-0 type PbZr0.52Ti0.48O3:xCoFe2O4 nanocomposite films Appl. Phys. Lett. 102, 122905 (2013); 10.1063/1.4799174

Exchange bias effect in multiferroic $\mathrm{CoCr} 2 \mathrm{O} 4 / \mathrm{Cr} 2 \mathrm{O} 3$ nanogranular system synthesized through a phase segregation route

J. Appl. Phys. 110, 053907 (2011); 10.1063/1.3632049

\section{Pure Metals - Ceramics} Alloys - Polymers in dozens of forms
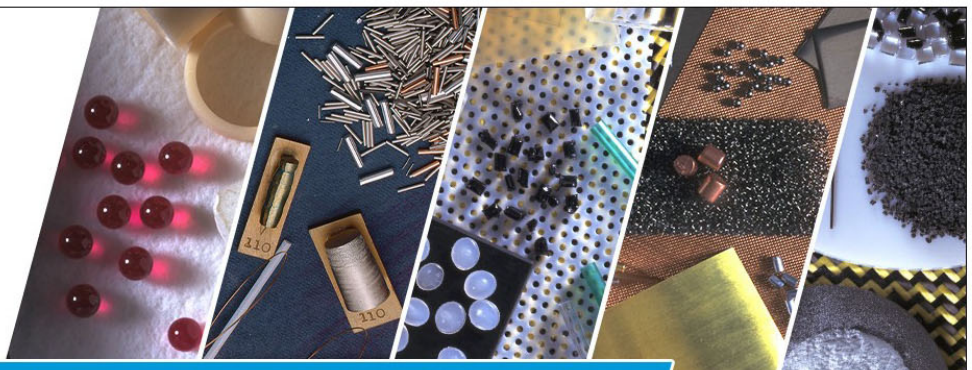


\title{
Ferromagnetic and multiferroic interfaces in granular perovskite composite $x \mathrm{La}_{0.5} \mathrm{Sr}_{0.5} \mathrm{CoO}_{3}-(1-\mathrm{x}) \mathrm{BiFeO}_{3}$
}

\author{
Javier H. Lohr, ${ }^{1}$ Carlos A. López, ${ }^{2, a), b)}$ Martín E. Saleta, ${ }^{1, b)}$ and Rodolfo D. Sánchez ${ }^{1, b), c)}$ \\ ${ }^{1}$ Centro Atómico Bariloche, Comisión Nacional de Energía Atómica, CONICET and Instituto Balseiro, \\ Universidad Nacional de Cuyo, 8400 San Carlos de Bariloche, RN, Argentina \\ ${ }^{2}$ INTEQUI, Universidad Nacional de San Luis, CONICET, Área de Química General e Inorgánica \\ "Dr. G.F.Puelles" - Facultad de Química, Bioquímica y Farmacia, Chacabuco y Pedernera, \\ 5700 San Luis, Argentina
}

(Received 26 April 2016; accepted 28 July 2016; published online 15 August 2016)

\begin{abstract}
Nanopowder of ferromagnetic $\mathrm{La}_{0.5} \mathrm{Sr}_{0.5} \mathrm{CoO}_{3}$ (LSCO) and multiferroic $\mathrm{BiFeO}_{3}$ (BFO) were synthesized by spray pyrolysis method. Different compositions of multiferroic $x$ LSCO- $(1-x)$ BFO composites were synthesized at $800^{\circ} \mathrm{C}$ for $2 \mathrm{~h}$. Scanning electron microscopy and energy dispersive spectroscopy elemental mapping were performed to study the morphology of composites. Ferri/ ferromagnetic responses above $T_{\mathrm{C}}$ (LSCO) are observed, which are associated with the interfaces LSCO/BFO. This interface presents a different behavior compared to the original perovskites, and the magnitude of the magnetization depends on $x$. Electrical DC conductivity as a function of temperature for LSCO nanopowder $(x=1)$ presents a different behavior than that reported in bulk material. For $x=1$ and 0.9, the model by Glazman and Matveev [Zh. Eksp. Teor. Fiz. 94, 332 (1988)] is proposed to describe the electrical conductivity. On the other hand, $x=0,0.1$, and 0.5 present a variable range hopping behavior. Complex impedance spectroscopy as a function of frequency indicates a pure resistive behavior for $x \geq 0.5$ compositions, while a complex resistivecapacitive behavior is observed for low $x$ values $(0,0.1)$. In these samples, low values of magnetoelectric coupling were measured with an AC lock-in technique. Published by AIP Publishing.

[http://dx.doi.org/10.1063/1.4960697]
\end{abstract}

\section{INTRODUCTION}

The synthesis and study of new multiferroic materials with coexistence of at least two ferroic orders as ferromagnetic (FM) and ferroelectric (FE) have increased strongly in the last few years. ${ }^{1}$ In particular, the search of magnetoelectric (ME) materials, where a dielectric polarization is induced with magnetic field or a magnetic polarization is driven by an electric field, is a matter of study in a new area for developing devices as memories. Its results are interesting because it would allow to combine ferroelectric data storage with magnetic reading. ${ }^{2}$ Several applications are quickly developing: magnetic field sensor (AC and DC), ME transformers, or gyrators as voltage gain devices and current sensors. ME effect is also useful in microwave devices like resonators, filters, and phase shifters. ${ }^{3}$

Single-phase multiferroic materials are rare. The coexistence between ferroelectricity and ferromagnetism can be uncoupled or coincident when magnetic order is developed. To increase the number of multiferroic materials, several fabrication alternatives have been performed as ferromagnetic/ ferroelectric superlattices ${ }^{4}$ or via simple solid solution routes as the synthesis of nanostructured composites or multiphase materials. ${ }^{5,6}$ Usually, in the composites formed by ferroelectric and ferromagnetic materials, the magnetoelectric coupling mechanisms can be based either on an interplay of

\footnotetext{
a) This research was conducted while visiting the Centro Atómico Bariloche. b) Members of the Research Career of CONICET.

${ }^{c}$ Author to whom correspondence should be addressed. Electronic mail: rodo@cab.cnea.gov.ar. Tel.: +54 294 4445158. Fax: +54 2944445269.
}

piezoelectricity and magnetostriction or on a charge carrier density modulation in the ferromagnetic phase that can induce a field effect. These two mechanisms can be superposed and it is indeed observed. ${ }^{7}$

In order to achieve that, it is convenient to use a ferroelectric (FE) material, like $\mathrm{BiFeO}_{3}(\mathrm{BFO})$ or $\mathrm{BaTiO}_{3}$ combined with a ferromagnetic (FM) material, similar to a colossal magnetoresistance (CMR) ferromagnet material or any ferro/ferrimagnetic oxide. ${ }^{8}$ Different magnetoelectric composite systems like $\mathrm{CoFe}_{2} \mathrm{O}_{4}-\mathrm{BaTiO}_{3}$ (CFO-BTO), ${ }^{9,10}$ (1-y) $\mathrm{BiFeO}_{3}-\mathrm{yNi}_{0.5} \mathrm{Cu}_{0.05} \mathrm{Zn}_{0.45} \mathrm{Fe}_{2} \mathrm{O}_{4},{ }^{11} \mathrm{xLa}_{0.625} \mathrm{Sr}_{0.375} \mathrm{MnO}_{3}-(1-\mathrm{x})$ $\mathrm{LuMnO}_{3},{ }^{12} x \mathrm{La}_{0.7} \mathrm{Sr}_{0.3} \mathrm{MnO}_{3}-(1-x) \mathrm{ErMnO}_{3},{ }^{13}$ and bilayers $\mathrm{La}_{0.7} \mathrm{Sr}_{0.3} \mathrm{MnO}_{3} / \mathrm{BaTiO}_{3}$ (LSMO/BTO), ${ }^{14} \mathrm{PbZr}_{0.52} \mathrm{Ti}_{0.48} \mathrm{O}_{3} /$ $\mathrm{La}_{0.67} \mathrm{Sr}_{0.33} \mathrm{MnO}_{3}{ }^{15}$ have been reported in the literature. Recently, the polarization study of $\mathrm{BiFeO}_{3} / \mathrm{La}_{0.5} \mathrm{Sr}_{0.5} \mathrm{CoO}_{3}$ films was reported. Using piezoresponse force microscopy (PFM) technique, the authors conclude that defect formation at the grain boundaries is responsible for the pinning centers that inhibit domain wall motion implying an asymmetric polarization-switching. ${ }^{16}$ Nonetheless, no study has been reported to date on $x \mathrm{La}_{0.5} \mathrm{Sr}_{0.5} \mathrm{CoO}_{3}-(1-x) \mathrm{BiFeO}_{3}$ solid nanocomposite. $\mathrm{BiFeO}_{3}$ is an auspicious single phase multiferroic compound. It shows an antiferromagnetic transition above room temperature, with a Néel temperature $T_{\mathrm{N}} \sim 640 \mathrm{~K}$, and a ferroelectric transition with a Curie temperature $T_{\mathrm{C}} \sim 1100 \mathrm{~K} .{ }^{17}$ The magnetoelectric coupling in BFO is weak, which means that the multiferroics property is not technological attractive in the single phase form, but it could be enhanced by preparing composites. On the other hand, a similar CMR material is related to $\mathrm{La}_{1-\mathrm{x}} \mathrm{Sr}_{\mathrm{x}} \mathrm{CoO}_{3}$ perovskite, which would be another interesting 
candidate for FE field-effect tuning. In the $x=0$ composition, the $\mathrm{Co}^{3+}$ cations are in the low-spin and nonmagnetic configuration at low temperature. For intermediate doping (LSCO: $x=0.5$ ), this cobalt perovskite has mix-valence and presents a metal behavior as the manganite; however, it is not a half metal as LSMO.$^{18}$ In particular, bulk LSCO presents a ferromagnetic transition at $T_{\mathrm{C}} \sim 250 \mathrm{~K}$, and below this temperature, the material presents a metallic behavior and itinerant ferromagnetism. Due to the $\mathrm{Sr}^{2+}$ doping, it presents different oxidation states of Co ions, $50 \% \mathrm{Co}^{3+}$ and $50 \% \mathrm{Co}^{4+} .{ }^{19}$

The nanostructuration of the materials can improve its magnetoelectric coupling due to the increment of the number of interfaces; with this idea, we synthesized the nanocomposite $x \mathrm{La}_{0.5} \mathrm{Sr}_{0.5} \mathrm{CoO}_{3}-(1-x) \mathrm{BiFeO}_{3}$.

Samples were characterized by transmission electron microscopy (TEM) and scanning electron microscopy (SEM) micrographs and energy dispersive spectroscopy (EDS), localized or elemental mapping. Magnetic, electric, and dielectric properties, as well as magnetoelectric coupling measurements, are presented and discussed in this work.

\section{EXPERIMENTAL}

BFO and LSCO powders have been prepared by spray pyrolysis method. ${ }^{20,21}$ The samples were deposited in a homemade spray-pyrolysis equipment described in Ref. 22. The acid solution used for the synthesis of $\mathrm{BFO}$ includes ferric nitrate $\left(\mathrm{Fe}\left(\mathrm{NO}_{3}\right)_{3} \cdot 9 \mathrm{H}_{2} \mathrm{O}\right)$ and bismuth nitrate $\left(\mathrm{Bi}\left(\mathrm{NO}_{3}\right)_{3} \cdot 5 \mathrm{H}_{2} \mathrm{O}\right)$ in appropriate molar proportions dissolved in a nitric acid/ water solution. The spray deposition has been made at $700^{\circ} \mathrm{C}$. The obtained powder has been annealed for $2 \mathrm{~h}$ at $800^{\circ} \mathrm{C}$ in air atmosphere. The LSCO powders have been prepared by the same method but using an acid solution of cobalt nitrate $\left(\mathrm{Co}\left(\mathrm{NO}_{3}\right)_{2} \cdot 6 \mathrm{H}_{2} \mathrm{O}\right)$, strontium carbonate $\left(\mathrm{SrCO}_{3}\right)$, and lanthanum nitrate $\left(\mathrm{La}\left(\mathrm{NO}_{3}\right)_{3} \cdot 6 \mathrm{H}_{2} \mathrm{O}\right)$. In this case, the spray deposition has been made at $800^{\circ} \mathrm{C}$ and the collected powder has been annealed at the same temperature under $\mathrm{O}_{2}$ atmosphere at atmospheric pressure. Nanocomposites of $x \mathrm{La}_{0.5} \mathrm{Sr}_{0.5} \mathrm{CoO}_{3}-$ $(1-x) \mathrm{BiFeO}_{3}$ have been prepared from $\mathrm{LSCO}$ and $\mathrm{BFO}$ nanopowders. Different amounts of powder have been mechanically mixed in an agate mortar in stoichiometric ratios in order to obtain different samples $(x=0.1,0.2,0.5,0.8$, and 0.9$)$. Afterwards, pellets were made for the different composites and annealed at $800^{\circ} \mathrm{C}$ under $\mathrm{O}_{2}$ flow for $2 \mathrm{~h}$. Structural characterization of samples was carried out by $\mathrm{X}$-ray powder diffraction (XRD) using a Philips PW1700 diffractometer $(\mathrm{Cu} \mathrm{K} \alpha$ radiation). Images of scanning electron microscopy (SEM) were collected with a FEI Nova Nano SEM 230 to observe morphology and perform a mapping of the atomic elements present in the samples. Transmission electron microscopy images were collected with a TEM Philips CM $200\left(\mathrm{LaB}_{6}\right.$ filament and acceleration voltage $200 \mathrm{kV}$ ), equipped with an ultratwin lens. The sample was milled in a mortar, and the particles obtained were dispersed in isopropyl alcohol. Drops of the colloidal suspension were placed on a grid of $\mathrm{Cu}$.

In order to characterize the electric and magnetic properties in these composites, we carried out different experiments. Magnetization-temperature dependence was measured in a commercial vibrating sample magnetometer (VSM) LakeShore
7300 between $100 \mathrm{~K}$ and $900 \mathrm{~K}$, as well as hysteresis magnetization loops at different temperatures. At $T=5 \mathrm{~K}$, a Quantum Design MPMS 5XS SQUID magnetometer was used. The electrical resistivity was measured in a four-probe homemade device in the temperature range $5 \mathrm{~K}<T<300 \mathrm{~K}$. The experimental device includes a Hewlett Packard 34420A nanovoltmeter, a Keithley 6221 DC current source, and a Lakeshore 330 temperature controller. For these DC transport measurements, composite pellets samples were cut in rectangular bars and the electrical contacts were made using silver paint. Complex electrical permittivity measurements were performed in a homemade device, the principal equipment being an Andeen Hagerling 2500A ultra precision capacitance bridge at $1 \mathrm{kHz}$. Samples were prepared like a parallel disc plate capacitor with approximately $1 \mathrm{~mm}$ of thickness. Complex impedance spectroscopy measurements in a range of frequency of $40 \mathrm{~Hz}-10 \mathrm{MHz}$ were carried out using an Agilent 4294A impedance analyzer. The transversal magneto-electric coefficient $\left(\alpha_{\mathrm{ME}}\right)$ measurements were carried out at room temperature in a homemade device employing a lock-in technique, ${ }^{23}$ with $20 \mathrm{Oe}$ of AC magnetic field excitation at $1 \mathrm{kHz}$ and static magnetic field bias between $\pm 7 \mathrm{kOe}$.

\section{RESULTS AND DISCUSSION}

\section{A. Structural and morphological characterization}

Figure 1 shows the $\mathrm{X}$-ray diffraction patterns of $x \mathrm{La}_{0.5} \mathrm{Sr}_{0.5} \mathrm{CoO}_{3}-(1-x) \mathrm{BiFeO}_{3}$ powder for $x=1,0.9,0.5$, and 0 . The XRD patterns, in particular, those $x=0$ and 1 , confirm that the synthesis method was successful. All XRD peaks could be clearly identified as of $\mathrm{La}_{0.5} \mathrm{Sr}_{0.5} \mathrm{CoO}_{3}$ (JCPDS Card No 00-048-0122) and $\mathrm{BiFeO}_{3}$ (JCPDS Card No 01-071-2494) along with some impurity peak of $\mathrm{Bi}_{25} \mathrm{FeO}_{40}$ (JCPDS Card No 00-046-0416). The $\mathrm{Bi}_{25} \mathrm{FeO}_{40}$ is

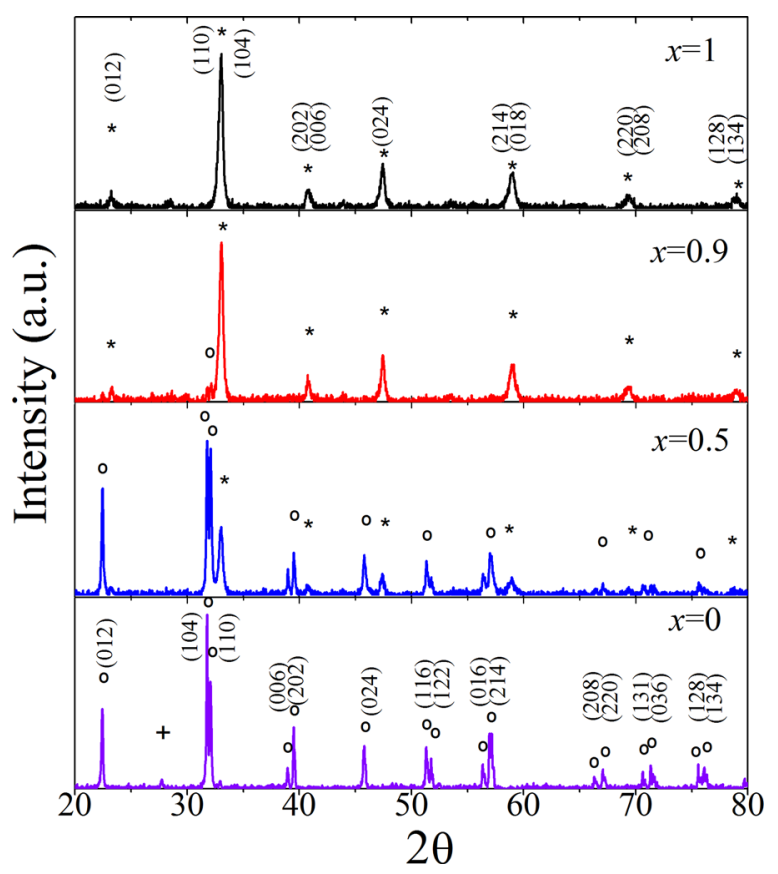

FIG. 1. X-ray diffraction patterns of $x \mathrm{La}_{0.5} \mathrm{Sr}_{0.5} \mathrm{CoO}_{3}-(1-x) \mathrm{BiFeO}_{3}$. (*), $(\mathrm{O})$, and $(+)$ indicate the $\mathrm{La}_{0.5} \mathrm{Sr}_{0.5} \mathrm{CoO}_{3}, \mathrm{BiFeO}_{3}$, and $\mathrm{Bi}_{25} \mathrm{FeO}_{40}$ expected diffraction peaks, respectively. 
a common spurious formed in BFO synthesis, but being a paramagnetic oxide, ${ }^{24,25}$ it should not interfere with the strong ferromagnetic response of the composite. The data in Fig. 1 show that the intensity of BFO peaks increases as $x$ decreases as expected.

Scanning electron microscopy (SEM) was used to observe the morphology of the samples. For the $x=0.5$ sample, a SEM image is presented in Fig. 2. Two groups of particles can be observed, one has small grain sizes, between $20 \mathrm{~nm}<d_{s}<100 \mathrm{~nm}$ (see inset), and the other group of particles presents sizes of $d_{s} \sim 1-10 \mu \mathrm{m}$. We collected data for EDS on different windows focusing on the different groups of particles. The EDS quantification shows that small particles are formed mainly by LSCO, while on the other hand large particles are mostly formed by BFO. EDS elemental mapping is presented in Fig. 3. The image in Fig. 3(a) is the visual SEM reference. In Fig. 3(b), violet colour represents rich regions associated with the $\mathrm{x}$-ray emission from the $\mathrm{K}$ shell energy level of Co element, which surround the large grain observed in the previous SEM image (Fig. 3(a)). Similar EDS mapping, due to the same amount of each ion, is obtained from the L shell energy levels of $\mathrm{Sr}$ and La elements, see Figs. 3(c) (red) and 3(d) (blue), respectively. On the contrary, the patterns observed in Figs. 3(e) (green) and 3(f) (yellow), which correspond to $\mathrm{Bi}(\mathrm{L})$ and $\mathrm{Fe}(\mathrm{K})$, respectively, show that these elements are located in the large grain. According to the information of EDS mapping of elements distribution, we conclude that the large grains with $d_{s}>1 \mu \mathrm{m}$ are formed by $\mathrm{BFO}$, while the smaller grains $\left(d_{s}<100 \mathrm{~nm}\right)$ correspond to the LSCO phase.

TEM images of the BFO were collected with different magnifications. Fig. 4(a) shows that the sample is constituted by grains with the size of tens of nanometers. The grains are crystalline as observed in the selected area electron diffraction (SAED) pattern. In Fig. 4(b), we indexed a SAED pattern of BFO in the expected space group with the cell parameters in agreement with the bibliography. ${ }^{26}$ Nanoparticles of BFO are observed in the image of Fig. 4(c). Crystalline planes can be observed in some particles.

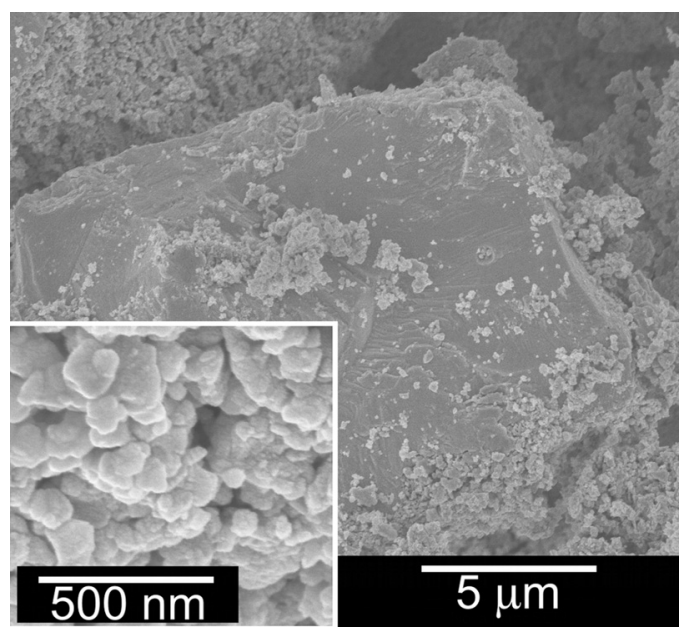

FIG. 2. SEM image of the $x=0.5$ sample: the inset shows in detail the region around a large grain.

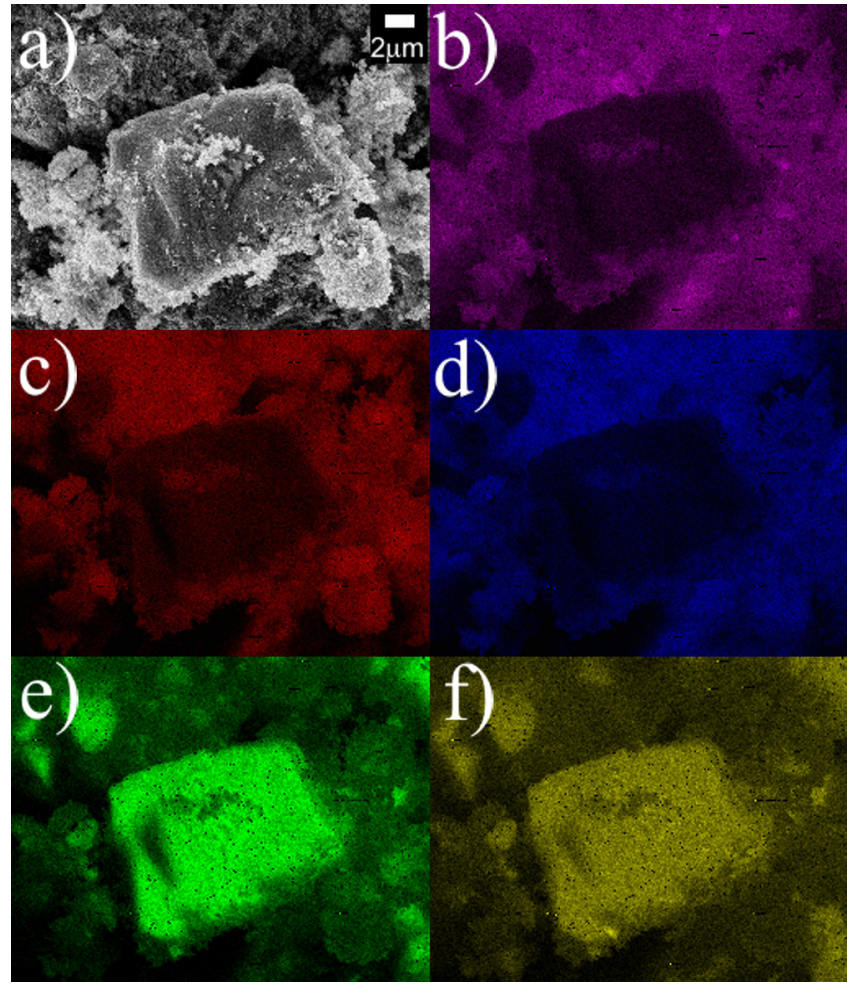

FIG. 3. EDS elemental mapping of the $x=0.5$ sample. (a) SEM reference image; atom mappings show different atom distributions: (b) Co-K line tuning (violet on-line); (c) Sr-L line (red on-line); (d) La-L line (blue on-line); (e) $\mathrm{Bi}-\mathrm{L}$ line (green on-line); and (f) $\mathrm{Fe}-\mathrm{K}$ line (yellow on-line), respectively.

\section{B. Magnetic properties}

Magnetization $(M)$ versus temperature $(T)$ curves at $H=100 \mathrm{Oe}$, after zero-field cooling (ZFC) condition, are shown in Fig. 5. In general, in all samples with $x>0, M$ increases to reach a maximum close to $230-250 \mathrm{~K}$, temperature depending on $x$. At high temperatures $(T>260 \mathrm{~K})$, an apparent paramagnetic behavior is observed. The critical temperature of the ferromagnetic order $\left(T_{\mathrm{C}}\right)$ has been determined as the minimum in $d M / d T$ vs $T$. This ferromagnetic transition temperature diminishes when the BFO fraction increases (see inset Fig. 5). This shift in the $T_{\mathrm{C}}$ can be associated with a hole doping effect where the charges are moved from the LSCO phase to BFO regions. Hysteresis loops were measured out at different temperatures. Fig. 6 presents the isothermal magnetization at $T=5 \mathrm{~K}$ for different $x$ values. For $x=1$, the magnetic saturation value $M_{\mathrm{S}} \sim 0.8 \mu_{\mathrm{B}} / \mathrm{Co}$ $(22 \mathrm{emu} / \mathrm{g})$ is lower than the reported for bulk samples $\left(1.7 \mu_{\mathrm{B}} / \mathrm{Co}\right) .{ }^{19}$ Usually, this fact associated with a magnetic dead layer presents in the shell of the nanoparticles. ${ }^{27,28}$ The inset in Fig. 6 presents the magnetic saturation at $5 \mathrm{~K}$ normalized for the LSCO mass in each sample, for which a constant value is expected. Nevertheless, in all cases the normalized $M_{\mathrm{S}}$ values are higher than the corresponding saturation for the LSCO composite end $(x=1)$, which may be attributable to another ferro/ferrimagnetic contribution.

On the other hand, isothermal magnetic curves at $T=300 \mathrm{~K}$ (above LSCO's $T_{\mathrm{C}}$ ) show an unexpected FM signal for intermediate compositions (Fig. 7). For clearness, we 


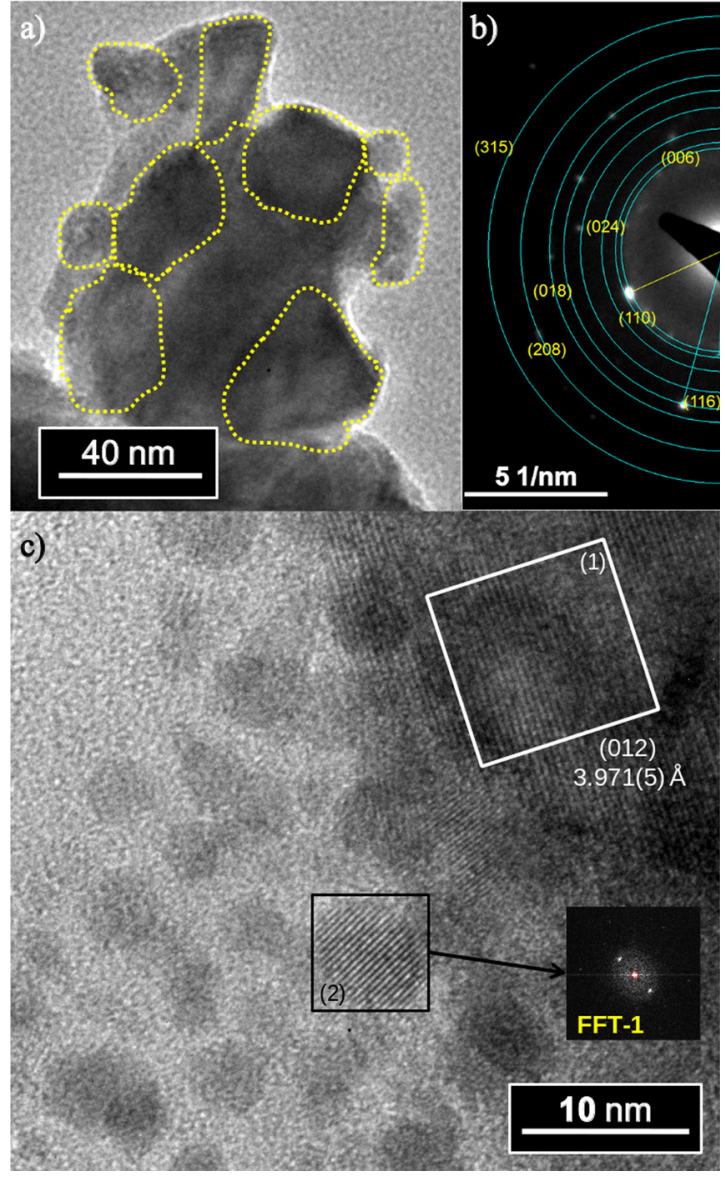

FIG. 4. (a) Low magnification image of BFO where particles of tens of nanometers are observed. (b) SAED pattern of BFO. The spots were indexed according to bibliographic information. (c) Image of particles with size under the tens nanometres. The separation of the planes of the box (1) is consistent with the separation of the planes of the family (012), of the hexagonal setting. Also, it is presented the FFT of the particle of the box (2).

show only three $x$ values at $300 \mathrm{~K}$. A linear behavior is observed in all the complete $H$ range measured for both ends, $x=0$ and 1 , respectively. In the BFO case, this is due to the AFM response $\left(T_{\mathrm{N}} \approx 640 \mathrm{~K}\right)$, whereas for $\mathrm{LSCO}$ $\left(T_{\mathrm{C}} \approx 250 \mathrm{~K}\right)$, the linearity is associated with the PM behavior. For intermediate $x$ values at $300 \mathrm{~K}$, the linear magnetic

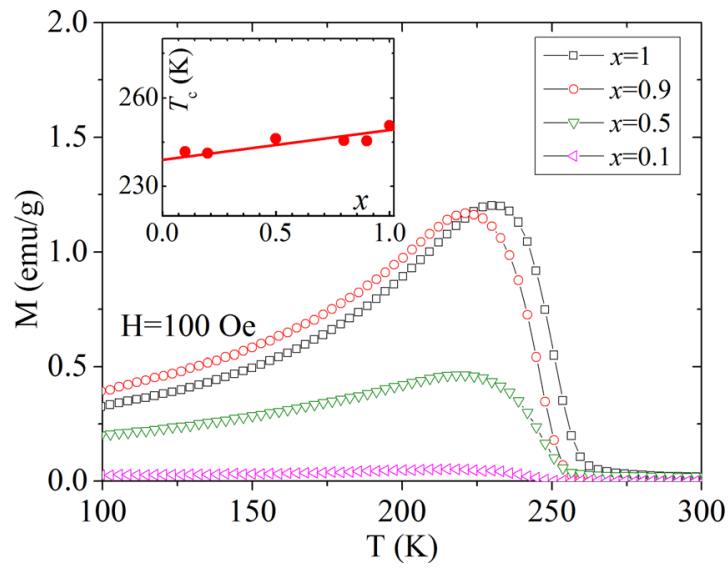

FIG. 5. Magnetization, after a ZFC process, as a function of temperature for an applied magnetic field of $100 \mathrm{Oe}$. The inset shows the variation of $T_{\mathrm{C}}$ (LSCO) with $x$.

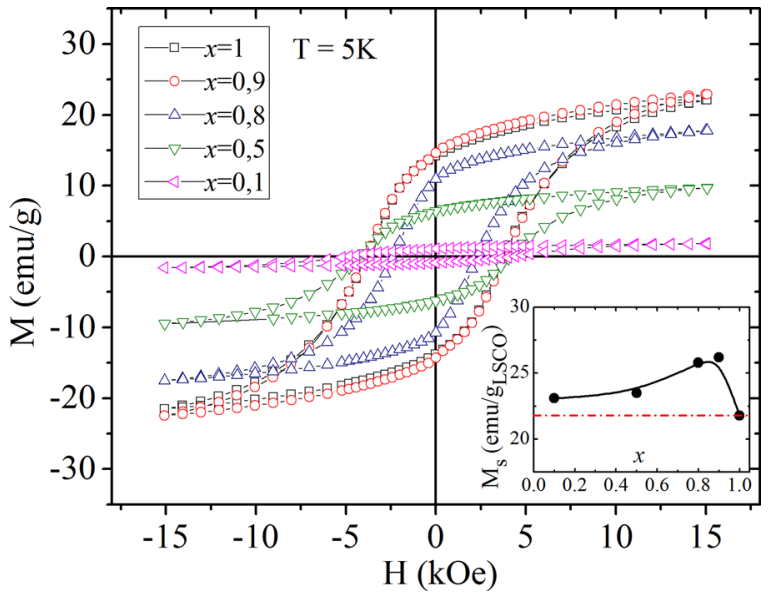

FIG. 6. Magnetic hysteresis loops at $5 \mathrm{~K}$ for $x \mathrm{LSCO}-(1-x) \mathrm{BFO}(\mathrm{x}=1,0.9$, $0.8,0.5$, and 0.1 ) composites. The inset shows $\mathrm{M}_{\mathrm{S}}$ (at $5 \mathrm{~K}$ ) normalized by the corresponding mass of LSCO at each value of $x$.

contribution of each phase (LSCO, BFO) is observed together with a FM loop. This linear behavior was fitted at high magnetic fields and subtracted to obtain the hysteresis loop. The magnetic saturation of this ferro/ferrimagnetic contribution $\left(M_{S} 300 \mathrm{~K}\right)$ is plotted in the inset of Fig. 7(a). It is interesting to remark that the magnetization saturation increases with $x$. In order to explain the origin of this additional contribution, we performed $M$ vs. $T$ experiments from room to high temperature $(T=900 \mathrm{~K})$. In Fig. $7(\mathrm{~b})$, we present the magnetization as a function of temperature for the $x=0.8$ composite. A change in slope is easily visible and associated to the AFM transition that occurs close to $T_{\mathrm{N}} \sim$ $640 \mathrm{~K}$ in the BFO material. In addition, a second transition is observed at higher temperatures, at $T \sim 700 \mathrm{~K}$. The ferro/ferrimagnetic contribution observed at room temperature together with this transition at $700 \mathrm{~K}$ is both associated with an additional magnetic phase, which is a product of the composite formation. This new phase should be formed and

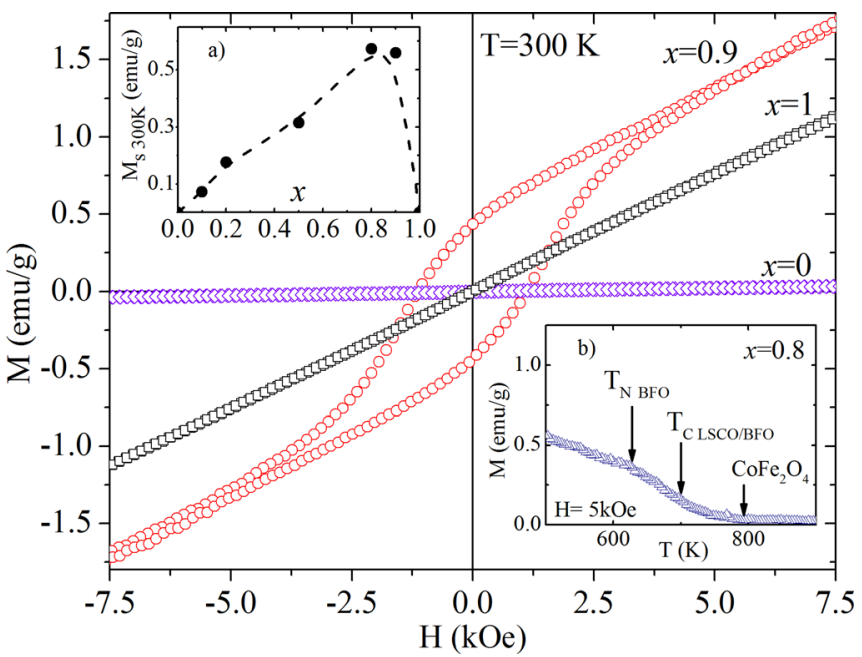

FIG. 7. Magnetization as a function of $\mathrm{H}$ at $300 \mathrm{~K}$, above $T_{\mathrm{C}}$ of $\mathrm{LSCO}$, for $x=1,0.9$, and 0 . Insets: (a) magnetic saturation after subtracting lineal contribution at high magnetic field. (b) Magnetization as a function of temperature at $5 \mathrm{kOe}$ for $0.8 \mathrm{LSCO}-0.2 \mathrm{BFO}$. The temperatures $T_{N}$ of BFO, $T_{C}$ of the interface $\mathrm{BFO} / \mathrm{LSCO}$ and $T_{C}$ of $\mathrm{CoFe}_{2} \mathrm{O}_{4}$ are indicated for references. 
located in the LSCO/BFO interface region. Considering the atomic elements that form the composites, one reasonable possibility could be the presence of an impurity of ferrimagnetic cubic spinel $\mathrm{Fe}_{3} \mathrm{O}_{4}\left(T_{C} \sim 850 \mathrm{~K}\right)$. This phase is usually responsible for higher saturation magnetization values in many cases. However, in our case and considering the observed Curie temperature observed, the presence of such phase is not consistent with our data. A second possibility can be the formation of $\mathrm{CoFe}_{2} \mathrm{O}_{4}$ in the interface. This spinel is ferrimagnetic with a $T_{\mathrm{C}}=793 \mathrm{~K}$ and $M_{\mathrm{s}}=80 \mathrm{emu} / \mathrm{g}$ in bulk. ${ }^{29}$ Using the bulk saturation magnetization and the saturation values obtained at room temperature, we have estimated the amounts of the spinel phase for each composite composition. We obtained between $0.1 \%$ and $0.8 \%$ of mass, which are undetectable for the XRD technique. By considering the value of the transition temperature, observed close to $700 \mathrm{~K}$, further possibility can be the formation in the interface of $\mathrm{Bi}_{1-x} \mathrm{La}_{\mathrm{x}} \mathrm{FeO}_{3}$. In this solid solution, the Neel temperature for a canted antiferromagnetic order goes from $T_{\mathrm{N}}=640 \mathrm{~K}$ for $x=0$ to $740 \mathrm{~K}$ for $x=1 .^{30}$

\section{Electrical properties}

The composites DC conductivity response, normalized to $280 \mathrm{~K}$ value $\left(\sigma / \sigma_{280 \mathrm{~K}}\right)$, is shown in Fig. 8 for different compositions. In all samples, the conductivity increases with the temperature in semiconductor materials. Nevertheless, when the data are plotted as $\ln \left(\sigma / \sigma_{280 \mathrm{~K}}\right)$ vs $1 / T$, the data do not follow a linear behavior for any value of $x$ expected for a thermal activation model. Alternatively, the data plotted as $\ln \left(\sigma / \sigma_{280 \mathrm{~K}}\right)$ vs $(1 / T)^{1 / 4}$ show a linear behavior for $x=0-0.5$ (see inset in Fig. 8). This temperature dependence indicates a possible conduction mechanism associated with disorder, where the electronic carriers are hopping under a variable and random potential between the cationic sites (variable range hopping or VRH). LSCO bulk is a ferromagnetic and metallic compound, presenting a typical itinerant ferromagnet electrical conductivity. The electrical resistivity presents quadratic temperature dependence below $T_{\mathrm{C}}$ and a linear

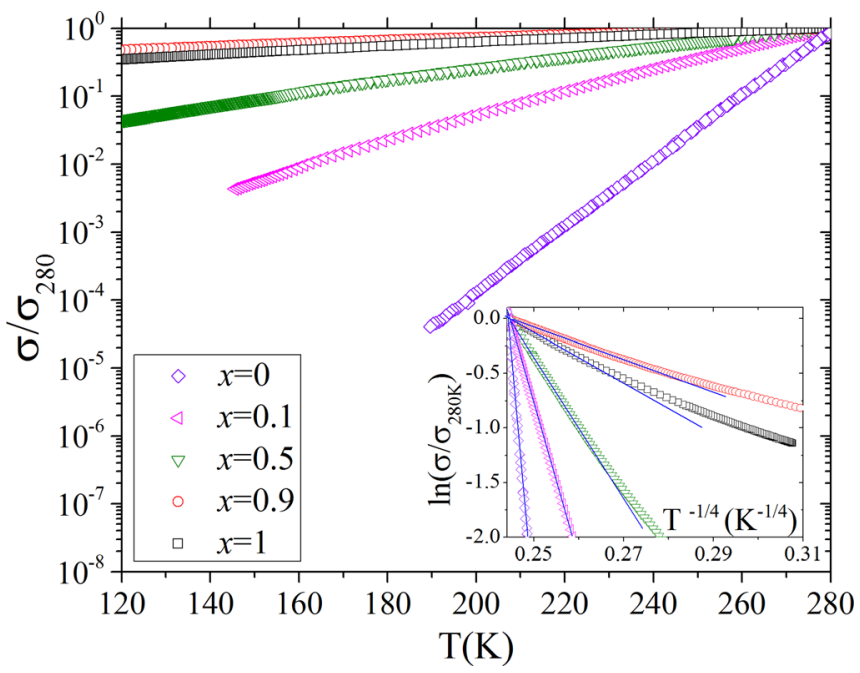

FIG. 8. DC conductivity normalized to the $280 \mathrm{~K}$ value $\left(\sigma / \sigma_{280 \mathrm{~K}}\right)$ as a function of temperature for $x \mathrm{LSCO}-(1-x) \mathrm{BFO}$ nanocomposite. The inset shows the agreement with a $\ln \left(\sigma / \sigma_{280 \mathrm{~K}}\right)$ vs. T ${ }^{-1 / 4} \mathrm{VRH}$ model for $x=0,0.1$, and 0.5 . behavior in the paramagnetic region as any normal metal. In our composites, and for high content of $\operatorname{LSCO}(x=0.9,1)$, the conductivity is different from the expected bulk behavior due to the grain boundaries. The LSCO nanostructured sample temperature behavior is opposite to the metallic bulk sample. ${ }^{19}$ Also, the absolute value of the conductivity at $T=280 \mathrm{~K}$ for nanostructured sample is two or three magnitude orders smaller than the bulk and metallic sample, $\sigma_{\text {nano }}$ $\sigma_{\text {Bulk }} \approx 10^{-2}-10^{-3}$. In similar nanostructured cases, other models have been proposed such as the Glazman and Matveev (GM) model, ${ }^{31}$ where the conductivity is mediated by intermediate localized states in the isolated barrier phase (shell of the particles ${ }^{27}$ ). This model is commonly used to describe metal-insulator-metal tunnel junctions. ${ }^{32}$ Excellent accord is found between the experimental conductivity and the GM model fit presented in Fig. 9. Resistance measurements with the magnetic field up to $9 \mathrm{~T}$ were performed for nanostructured LSCO. The inset of Fig. 9 shows the MR \% $(100[\mathrm{R}(\mathrm{H})-\mathrm{R}(0)] / \mathrm{R}(0))$ at different temperatures; the MR value for $9 \mathrm{~T}$ at $4.2 \mathrm{~K}$ is three times higher than the bulk material value. ${ }^{19}$

Figure 10 presents the conductivity values at $280 \mathrm{~K}$ for different molar fractions $(x)$. Two $\sigma$ regimens are observed. For $x=0$ (BFO), the system is completely insulator and suddenly its conductivity increases seven orders of magnitude when small amounts of LSCO phase are introduced $(0<x<0.2)$. From $x=0.2$ to $x=1$, the electrical conductivity increases five orders of magnitude to reach the conductivity of the pure LSCO. Considering the presence of these two different phases (BFO and LSCO) with very different conductivities, we propose a single conductance (or resistance) arrays. To describe $x<0.2$ compositions, we propose a parallel conductance array (or two resistances in series) and for the other regime, $x>0.2$, conductances in series are proposed (or two parallel resistances). By applying the effective-medium approximation (EMA) model, considering two phases with different conductivities, an homogeneous distribution and 3D system, the volume fraction percolation

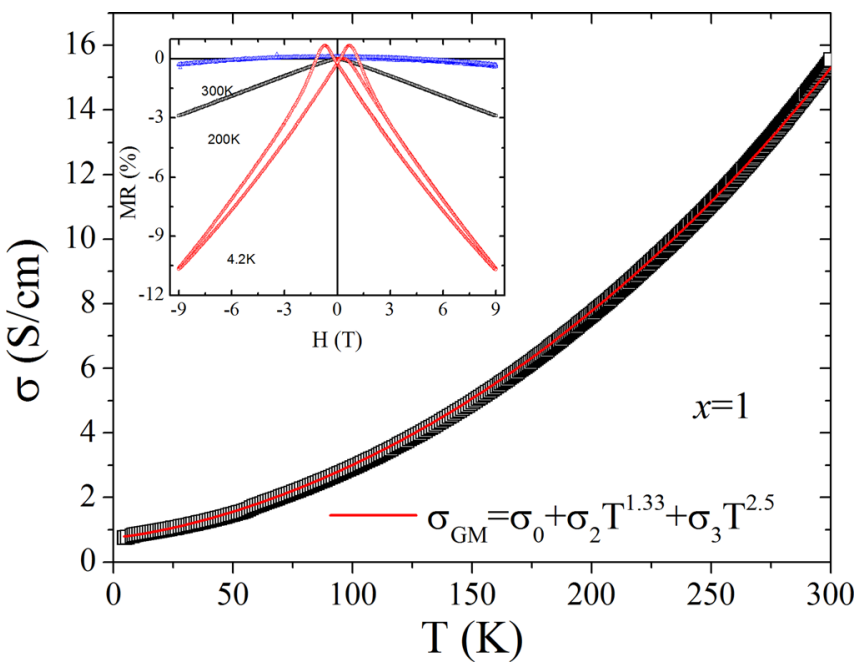

FIG. 9. DC conductivity as a function of temperature for LSCO nanostructured sample $(x=1)$. The line (red online) shows the fit with the GlazmanMatveev model which describes inelastic hopping via chains of two and three localized states. 


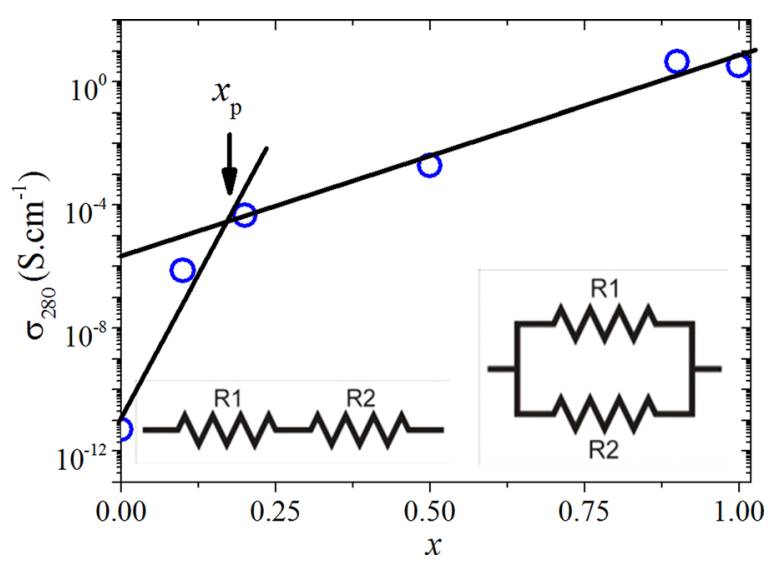

FIG. 10 . DC conductivity at $\mathrm{T}=280 \mathrm{~K}$ for $x \mathrm{LSCO}-(1-x) \mathrm{BFO}$ nanocomposite with different $x$ values. The solid lines are only guides to the eye, indicating the different regimes.

threshold is predicted to be $f_{\mathrm{p}}{ }^{*}=1 / 3 .^{33}$ For a particular case under study, taking the unit cell volume of the each end member $\left(V_{\text {cell }}(\mathrm{BFO})=374.3 \AA^{3}\right.$ and $V_{\text {cell }}(\mathrm{LSCO})=338.4$ $\AA^{3}$ ) and taking the different molecular weights between the compounds $\mathrm{W}(\mathrm{LSCO}) / \mathrm{W}(\mathrm{BFO}) \sim 0.7$, the expected percolation threshold expressed as molar fraction is $x_{p}{ }^{*} \sim 0.36$. This value is somewhat higher than the observed cross-over $\left(x_{p} \sim\right.$ 0.2 ) in Fig. 10. One possible reason to explain this shift can be the different sizes of the LSCO particles with respect to the large BFO. In other words, it can be a consequence of nanostructuration, where the high conductivity nanograins of LSCO surround the BFO insulator micrograins (see Fig. 3) and form percolation paths with a low molar fraction. This value is approximately half the estimated value for a homogeneous two-phase (metallic and insulator) distribution.

Complex electrical permittivity measurements could only be performed in samples with small losses as the insulating samples $x=0$ and $x=0.1$, whose data are shown in Fig. 11. For $\mathrm{BiFeO}_{3}(x=0)$, we measured a low temperature intrinsic value of $\varepsilon^{\prime} \sim 50$ which is in concordance with that reported in literature. ${ }^{34}$ With the incorporation of the LSCO

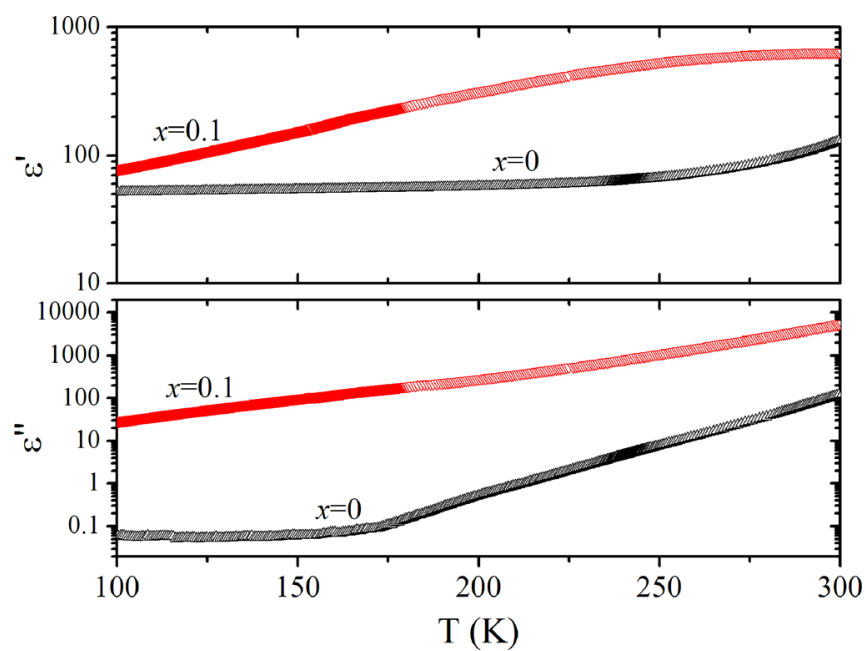

FIG. 11. Electric permittivity $\left(\varepsilon^{\prime}\right)$ and electrical loss $\left(\varepsilon^{\prime \prime}\right)$ constants at $1 \mathrm{kHz}$ as a function of temperature for $x=0$ and 0.1 . No anomalies are detected in the measured temperature range. phase, a loss increment $\left(\varepsilon^{\prime \prime}\right)$ is expected and experimentally observed (see comparison between $x=0.1$ and $x=0$ in Fig. 11). Any anomaly associated with a ferroelectric transition or to the magnetic transition of LSCO was not observed.

Complex impedance spectroscopy measurements (Z) were carried out for different composites $x=0,0.1,0.5$, and 0.9 at room temperature within a frequency range of $40 \mathrm{~Hz}-10 \mathrm{MHz}$ (Fig. 12). Composites with $x=0.9$ and 0.5 show a pure resistive behavior (phase $=0$ ) in the studied range. For the low resistance end, $x \geq 0.9$, an inductive contribution appears at high frequencies, which we attribute to our experimental limitations. The $x=0.1$ sample presents an interesting "steps-likes" dependence in the phase parameter, a behavior that is also observed in other oxides. ${ }^{35,36}$ At low frequencies, the sample presents a resistive plateau behavior (phase $=0$ ) and when the frequency is increased, beyond $200 \mathrm{~Hz}$, the phase parameter increases its negative values, showing a step-like or capacitive contribution. A second step is observed due to a second capacitive contribution around $200 \mathrm{kHz}$. In the BFO $(x=0)$, the capacitive contribution at low frequencies is more prominent and other contributions are observed at high frequencies.

The expected complex Cole-Cole plot, $\operatorname{Re}[\mathrm{Z}]$ vs -Im[Z], for the $x=0.1$ sample should consist of two superimposed semicircles. In this kind of plots, a semicircle describes a parallel resistance-capacitor electrical circuit. The experimental data and their fit are presented in Fig. 13, where we show that there are two characteristic times in the system coming from each set of the RC circuits. The possible explanation about the physical origin of these two characteristic times is that one is associated with the grain boundaries and other one is related to the bulk behavior. The inset in Fig. 13 shows the complex diagram for $x=0$ and also presents two characteristic times like the $x=0.1$ sample; however, the extremely high resistance values prevent the observation of the complete semicircle.

In order to complete the characterization of the insulator samples, we performed polarization vs. electric field experiments using a homemade Sawyer-Tower circuit. Unfortunately,
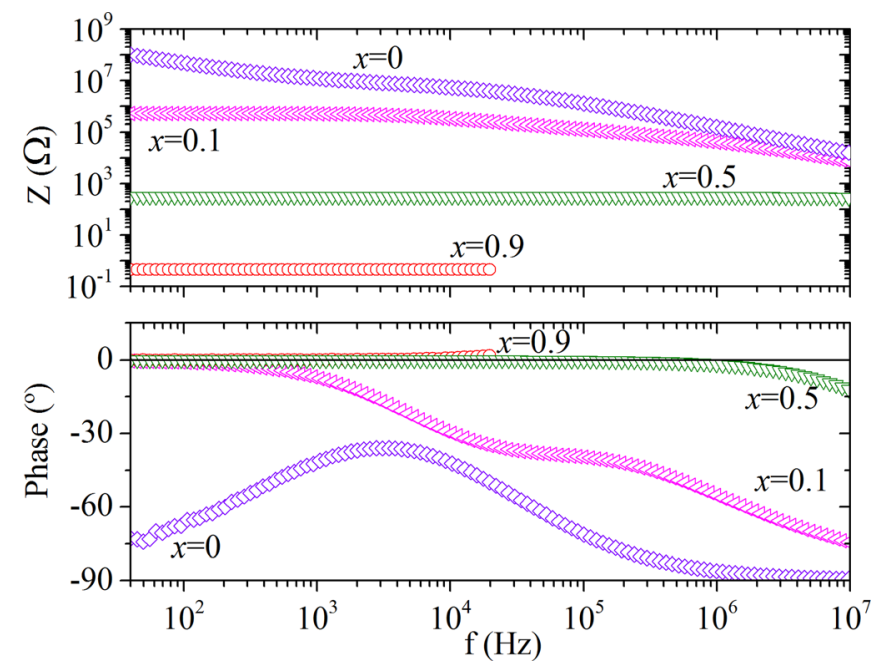

FIG. 12. Modulus $\mathrm{Z}$ and phase of complex impedance as a function of frequency for different $x \mathrm{LSCO}-(1-x) \mathrm{BFO}$ composites. 


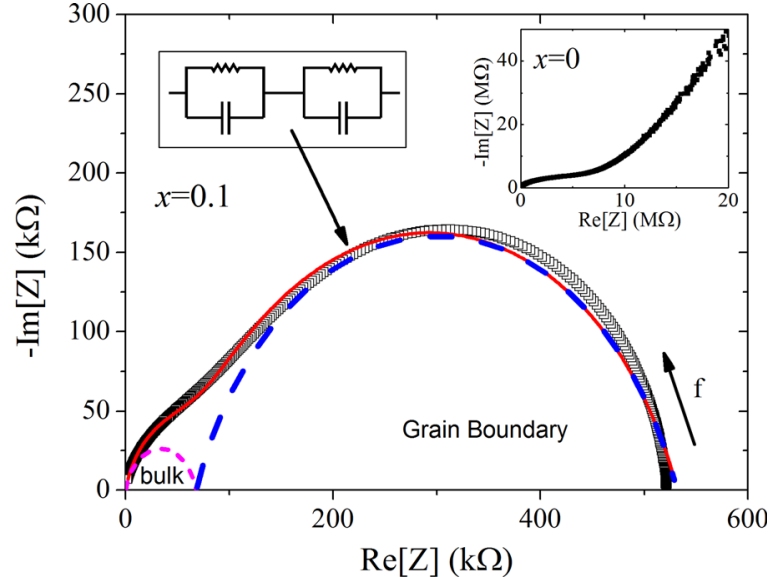

FIG. 13. Real vs. imaginary component of complex electric impedance from a measurement in the range of frequency $40 \mathrm{~Hz}-10 \mathrm{MHz}$ for the sample with $x=0.1$. The red line is a fit to the $2 \mathrm{RC}$ parallel model. Dashed lines indicate two contributions to the impedance. The inset shows the BFO results for the same experiment.

we have not detected a feasible ferroelectric hysteresis curve at room temperature. The principal reason is due to the loss currents by the high AC conductivity $\left(\sigma_{\mathrm{ac}} \approx 10^{-8} \Omega^{-1} \mathrm{~cm}^{-1}\right)$ of the samples. For this particular case, we applied $1.5 \mathrm{kV}$ between $1 \mathrm{~mm}$ of thickness, which gives a maximum electric field of $15 \mathrm{kV} \mathrm{cm}^{-1}$ as our experimental limit (see supplementary material Fig. S1 for $x=0$ sample). Other authors, e.g., Lu et al., ${ }^{34}$ have obtained ferroelectrics loops of BFO single crystals, below $200 \mathrm{~K}$ and applying electric field of $20 \mathrm{kV} \mathrm{cm}^{-1}$.

Finally, the magnetoelectric (ME) characterization of the most insulating composite sample $(x=0.1)$ has been performed. In order to compare the ME response with a typical composite, we also synthesized a ceramic composite of $0.5 \mathrm{CoFe}_{2} \mathrm{O}_{4}-0.5 \mathrm{BaTiO}_{3}$ via Solid State Reaction Method (SSRM). The magnetoelectric transversal coefficient $\left(\alpha_{\mathrm{ME}}\right)$ of both samples is presented in Fig. 14. The $\alpha_{\mathrm{ME}}$ value for $0.1 \mathrm{LSCO}-0.9 \mathrm{BFO}$ is $0.710^{-3} \mathrm{mV} \mathrm{cm}^{-1} \mathrm{Oe}^{-1}$, which is three orders of magnitude less than the reference composite used. In order to compare with the data of the bibliography, different materials that include films and ceramic composites

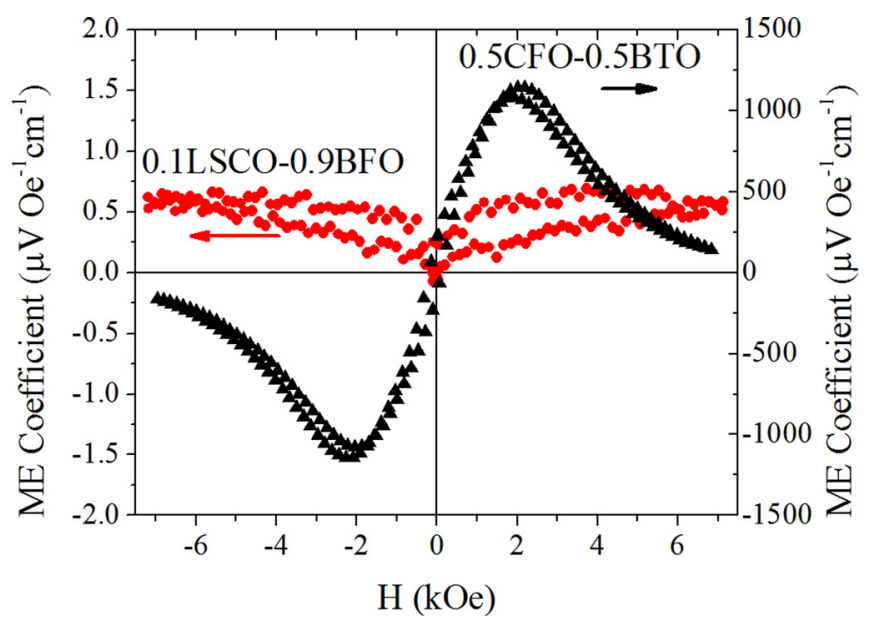

FIG. 14. Magneto-electric coefficient as a function of DC magnetic field. Circles correspond to the $x=0.1$ composite and triangles to a reference compound $0.5 \mathrm{CoFe}_{2} \mathrm{O}_{4}-0.5 \mathrm{BaTiO}_{3}$. fabricated by solid state reaction or by sol-gel routes forming nanopowders are discussed. According to the magnetoelectric response, the samples can be divided in three groups classified by the strength of coupling. In the first group, we include high strength ME coupling materials as the TerfenolD/PVDF (polyvinylidenedifluoride) composite, which has $\alpha_{\mathrm{ME}}=1430 \mathrm{mV} \mathrm{cm}{ }^{-1} \mathrm{Oe}^{-137}$ Other composite that may be included in this group is the $\mathrm{Bi}_{4} \mathrm{Ti}_{3} \mathrm{O}_{12} / \mathrm{Bi}_{5} \mathrm{Ti}_{3} \mathrm{FeO}_{15}$ films with $\alpha_{\mathrm{ME}}=78 \mathrm{mV} \mathrm{cm}^{-1} \mathrm{Oe}^{-1} \cdot{ }^{38}$ Ferromagnetic/ferroelectric composite materials are formed by two kinds of single phases, which can be independently or ME coupled between them. The ME effect is a new functionality originated by the interaction between the phases. The accepted coupling picture is the combination of piezoelectric effect of ferroelectric phase/layer (composite/film) with magnetostriction effect of ferromagnetic phase/layer. A strong coupling between ferroelectric and ferromagnetic phases/layers can helpful to obtain greater magnetoelectric signal. A second consideration is the formation of intermediate interface between the contact of both phases/layers, which can perform an excellent ME coupling.

The second group associates with moderate $\mathrm{ME}$ coupling strength materials. Composites like $x \mathrm{Ba}_{0.95} \mathrm{Sr}_{0.05} \mathrm{TiO}_{3}-$ $(1-x) \mathrm{BiFe}_{0.9} \mathrm{Gd}_{0.1} \mathrm{O}_{3}$, prepared by SSRM, give $\alpha_{\mathrm{ME}}=1.67 \mathrm{mV}$ $\mathrm{cm}^{-1} \mathrm{Oe}^{-1} .39$ In this group, we also include our $0.5 \mathrm{CoFe}_{2} \mathrm{O}_{4}-$ $0.5 \mathrm{BaTiO}_{3}$ reference composite $(\mathrm{SSRM})$ with $\alpha_{\mathrm{ME}}=1.1 \mathrm{mV}$ $\mathrm{cm}^{-1} \mathrm{Oe}^{-1}$ and the previously published $\alpha_{\mathrm{ME}}=0.52 \mathrm{mV}$ $\mathrm{cm}^{-1} \mathrm{Oe}^{-1}$ about the same composite formed by nanopowders. ${ }^{40}$ It is interesting to note how $\alpha_{\mathrm{ME}}$ decreases when the grain size is reduced. To have a realistic picture in this complex scenario, other factors should also take into account such as anisotropy, domain structure, interface defects, grain boundaries, and/or charge pooling capacity that can affect notably the ME coupling. For example, in the laminate composite $\mathrm{La}_{0.7} \mathrm{Ba}_{0.3} \mathrm{MnO}_{3}-\mathrm{BaTiO}_{3}\left(\alpha_{\mathrm{ME}}=0.65 \mathrm{mV} \mathrm{cm}{ }^{-1} \mathrm{Oe}^{-1}\right)$, the moderate ME coupling is attributed to the low density, caused by differences in thermal expansion, as well as the grain size effect which can be controlled by the synthesis method. ${ }^{41}$ Diffusion process directly affects the internal residual stresses and chemically inhomogeneous regions.

Finally, the III group (low ME coupling strength values) are formed by composites as $0.85 \mathrm{Bi}_{0.95} \mathrm{Dy}_{0.05} \mathrm{FeO}_{3}-0.15 \mathrm{BaTiO}_{3}{ }^{42}$ that presents extremely low values $\left(\alpha_{\mathrm{ME}}=1.8 \quad 10^{-3} \mathrm{mV}\right.$ $\left.\mathrm{cm}^{-1} \mathrm{Oe}^{-1}\right)$. The nanocomposite samples studied in this work are included in this group. The low ME coupling observed can be due to different possibilities. One of them is the diminution of piezoelectric effect when the grain size is reduced. It has been observed by Lei et al. ${ }^{43}$ in the nanostructured and polycrystalline BFO films. The piezoelectric coefficient is reduced ten times when the grain size is reduced from 150 to $85 \mathrm{~nm}$. Other explanation might be the high electrical leakages in polycrystalline composite samples due to the presence of a high conductivity phase. The high voltage source used in these kinds of experiments is limited by the current compliance, limit that is easily reached with the high electric leakages and consequently avoid to apply high electric field and to reach the saturation of the polarization to reach a complete parallel orientation of the ferroelectric domains. 


\section{CONCLUSIONS}

We prepared different compositions of multiferroic $x \mathrm{La}_{0.5} \mathrm{Sr}_{0.5} \mathrm{CoO}_{3}-(1-x) \mathrm{BiFeO}_{3}(x \mathrm{LSCO}-(1-x) \mathrm{BFO})$ composites. The starting materials were ferromagnetic LSCO and multiferroic BFO nano powders, which were synthesized by spray pyrolysis method at $800^{\circ} \mathrm{C}$. X-ray diffraction and EDS-SEM techniques confirmed the desired phases and the expected ratio compositions. The SEM morphology and EDS elemental mapping evidenced larger BFO grains (composed by nanocrystals observed by TEM) surrounded by LSCO nanocrystals.

The LSCO Curie temperature smoothly decreases when the BFO ratio increases. A magnetic impurity phase, undetectable by XRD, was observed in magnetization experiments. We assume that this ferri/ferromagnetic phase is located in the interface of LSCO/BFO and it is formed during the annealing process. The chemical composition of this new phase can be close to the spinel $\mathrm{CoFe}_{2} \mathrm{O}_{4}$. Note that this additional phase is not present in the composite ends (LSCO or BFO).

Electrical conductivity showed that a variable range hopping mechanism dominates for small $x$ compositions, whereas for large $x$ (rich in LSCO), the conductivity is described by a Glazman-Matveev mechanism, due to the nanostructure of the LSCO phase. Also, for these compositions, an important coupling between the conductivity and the magnetic field was observed which is larger than the one for bulk LSCO. A phenomenological description of parallel and series resistances can be used to describe the conductivity at room temperature with the change of the LSCO/BFO phase ratio. A percolation threshold was determined close to $x \sim 0.2$. No anomalies have been observed in the electrical permittivity, but important changes in its magnitude and temperature dependence are observed when 10 mol. \% of LSCO is added to the BFO phase. The complex electric impedance measurements at room temperature indicate that composites with $x=0.9$ and 0.5 have an ohmic resistive behavior, whereas on the other hand, $x=0.1$ and 0 present a resistive-capacitive parallel behavior. A model with two RC parallel circuits was proposed, which are associated with the bulk and grain boundary contributions. We associate the low $\mathrm{ME}$ coupling observed in this nanocomposite to the weak ferroelectric polarization. It may be due to the high electrical leakages, which limit the experimental applied electric field, or by defects that pinning the ferroelectric domains avoiding their parallel orientation. Finally, we have shown that the low-temperature of the spray-pyrolysis synthesis provides an alternative route for novel integrated nanocomposite materials and devices.

\section{SUPPLEMENTARY MATERIAL}

See supplementary material Figure $\mathrm{S} 1$ for ferroelectric hysteresis loop at room temperature for $\mathrm{BiFeO}_{3}$ (pressed nanoparticles sample) with $1 \mathrm{~mm}$ of thickness. Maximum electric field: $15 \mathrm{kV} \mathrm{cm}^{-1}$. The loop was measured using a homemade Sawyer-Tower circuit.

\section{ACKNOWLEDGMENTS}

R.D.S. thanks support from Consejo Nacional de Investigaciones Científicas y Técnicas (CONICET), PIP
0490, the Agencia Nacional de Promoción Científica y Tecnológica (ANPCyT), PICT-2011-752, and the Secretaría de Ciencia, Tecnología y Planificación de la Universidad Nacional de Cuyo (SECTyP-UNCuyo), Project 06/C456. We gratefully acknowledge Virginia Tognolli for technical assistance, Horacio Troiani for assistance in the TEM microscopy, and Gabriela Aurelio for critical reading of the manuscript. J.L. thanks CONICET for a fellowship.

${ }^{1}$ D. I. Khomskii, J. Magn. Magn. Mater. 306, 1 (2006).

${ }^{2}$ M. Bibes and A. Barthélémy, Nat. Mater. 7, 425 (2008).

${ }^{3}$ C. W. Nan, M. I. Bichurin, S. Dong, D. Viehland, and G. Srinivasan, J. Appl. Phys. 103, 031101 (2008).

${ }^{4}$ P. Murugavel, D. Saurel, W. Prellier, Ch. Simon, and B. Raveau, Appl. Phys. Lett. 85, 4424 (2004).

${ }^{5}$ J. Beltran-Huarac, R. Martinez, and G. Morell, J. Appl. Phys. 115, 084102 (2014).

${ }^{6}$ J. S. Andrew, J. D. Starr, and M. A. K. Budi, Scr. Mater. 74, 38 (2014).

${ }^{7}$ H. J. A. Molegraaf, J. Hoffman, C. A. F. Vaz, S. Gariglio, D. van der Marel, C. H. Ahn, and J. M. Triscone, Adv. Mater. 21, 3470 (2009).

${ }^{8}$ S. H. Kshirsagar, A. N. Tarale, S. R. Jigajeni, D. J. Salunkhe, S. B. Kulkarni, and P. B. Joshi, J. Mater. Sci.: Mater. Electron. 27, 375 (2016).

${ }^{9}$ A. Hanumaiah, T. Bhimasankaram, S. V. Suryanarayana, and G. S. Kumar, Bull. Mater. Sci. 17, 405 (1994).

${ }^{10}$ H. Zheng, J. Wang, S. E. Lofland, Z. Ma, L. Mohaddes-Ardabili, T. Zhao, L. Salamanca-Riba, S. R. Shinde, S. B. Ogale, F. Bai, D. Viehland, Y. Jia, D. G. Schlom, M. Wuttig, A. Roytburd, and R. Ramesh, Science 303, 661 (2004).

${ }^{11}$ S. C. Mazumdar, M. N. I. Khan, Md. Fakhrul Islam, and A. K. M. Akther Hossain, J. Magn. Magn. Mater. 390, 61 (2015).

${ }^{12}$ G. B. Song, J. S. Amaral, V. S. Amaral, and A. L. Kholkin, J. Eur. Ceram. Soc. 27, 3941-3945 (2007).

${ }^{13}$ P. Dey, T. K. Nath, M. L. N. Goswami, and T. K. Kundu, Appl. Phys. Lett. 90, 162510 (2007).

${ }^{14}$ T. X. Li, M. Zhang, F. J. Yu, Z. Hu, K. S. Li, D. B. Yu, and H. Yan, J. Phys. D: Appl. Phys. 45, 085002 (2012).

${ }^{15}$ S. Dussan, A. Kumar, J. F. Scott, and R. S. Katiyar, Appl. Phys. Lett. 96, 072904 (2010).

${ }^{16}$ P. R. Choudhury, J. Parui, S. Chiniwar, and S. B. Krupanidhi, Solid State Commun. 208, 15 (2015).

${ }^{17}$ P. Fischer, M. Polomska, I. Sosnowska, and M. Szymanski, J. Phys. C: Solid State Phys. 13, 1931 (1980); J. Wang, J. B. Neaton, H. Zheng, V. Nagarajan, S. B. Ogale, B. Liu, D. Viehland, V. Vaithyanathan, D. G. Schlom, U. V. Waghmare, N. A. Spaldin, K. M. Rabe, M. Wuttig, and R. Ramesh, Science 299, 1719 (2003).

${ }^{18}$ J. H. Park, E. Vescovo, H. J. Kim, C. Kwon, R. Ramesh, and T. Venkatesan, Nature 392, 794 (1998).

${ }^{19}$ D. Samal and P. S. Anil Kumar, J. Phys.: Condens. Matter 23, 016001 (2011).

${ }^{20}$ S. Che, O. Sakurai, K. Shinozaki, and N. Mizutani, J. Aerosol Sci. 29, 271 (1998).

${ }^{21}$ K. Okuyama and I. W. Lenggoro, Chem. Eng. Sci. 58, 537 (2003).

${ }^{22}$ M. E. Saleta, M. Granada, J. Curiale, R. Benavides, H. E. Troiani, and R. D. Sánchez, J. Phys.: Condens. Matter 23, 275301 (2011).

${ }^{23}$ G. V. Doung, R. Groessinger, M. Schoenhart, and D. Bueno-Basques, J. Magn. Magn. Mater. 316, 390 (2007).

${ }^{24}$ A. Maître, M. François, and J. C. Gachon, J. Phase Equilib. Diffus. 25, 59 (2004).

${ }^{25}$ R. Köferstein, T. Buttlar, and S. G. Ebbinghaus, J. Solid State Chem. 217, 50 (2014).

${ }^{26}$ F. Kubel and H. Schmid, Acta Crystallogr. B46, 698 (1990).

${ }^{27}$ J. Curiale, M. Granada, H. E. Troiani, R. D. Sánchez, A. G. Leyva, P. Levy, and K. Samwer, Appl. Phys. Lett. 95, 043106 (2009).

${ }^{28}$ M. A. López-Quintela, L. E. Hueso, J. Rivas, and F. Rivadulla, Nanotechnology 14, 212 (2003).

${ }^{29}$ B. D. Cullity and C. D. Graham, Introduction to Magnetic Materials, 2nd ed. (Wiley-IEEE Press, 2008), p. 183, ISBN: 978-0-471-47741-9.

${ }^{30}$ V. V. Lazenka, A. F. Ravinski, I. I. Makoed, J. Vanacken, G. Zhang, and V. V. Moshchalkov, J. Appl. Phys. 111, 123916 (2012).

${ }^{31}$ L. I. Glazman and K. A. Matveev, Zh. Eksp. Teor. Fiz. 94, 332 (1988); L. I. Glazman and K. A. Matveev, Sov. Phys. JETP 67, 1276 (1988). 
${ }^{32}$ Y. Xu, D. Ephron, and M. R. Beasley, Phys. Rev. B 52, 2843 (1995).

${ }^{33}$ D. J. Bergman and D. Stroud, "Physical properties of macroscopically inhomogeneous media," Solid State Phys. 46, 147-269 (1992).

${ }^{34}$ J. Lu, A. Günther, F. Schrettle, F. Mayr, S. Krohns, P. Lunkenheimer, A. Pimenov, V. D. Travkin, A. A. Mukhin, and A. Loidl, Eur. Phys. J. B 75, 451 (2010).

${ }^{35}$ M. Vollman and R. Waser, J. Am. Ceram. Soc. 77, 235 (1994).

${ }^{36}$ J. Fleig and J. Maier, J. Electrochem. Soc. 145, 2081 (1998).

${ }^{37}$ K. Mori and M. Wuttig, Appl. Phys. Lett. 81, 100 (2002).

${ }^{38}$ J. Chen, Y. Bai, C. Nie, and S. Zhao, J. Alloys Compd. 663, 480 (2016).
${ }^{39}$ M. J. Miah, M. N. I. Khan, and A. K. M. Akther Hossain, J. Magn. Magn. Mater. 401, 600 (2016).

${ }^{40}$ V. V. Shvartsman, F. Alawneh, P. Borisov, D. Kozodaev, and D. C. Lupascu, Smart. Mater. Struct. 20, 075006 (2011).

${ }^{41}$ J. L. Clabel, H., F. A. Ferri, F. L. Zabotto, V. A. G. Rivera, I. C. Nogueira, D. Garcia, O. F. de Lima, E. R. Leite, M. A. Pereira-da-Silva, and C. A. Cardoso, J. Magn. Magn. Mater. 407, 160 (2016).

${ }^{42}$ A. Sathiya Priya, I. B. Shameem Banua, and S. Anwar, Mater. Lett. 142, 42 (2015).

${ }^{43}$ T. Lei, W. Cai, C. Fu, H. Ren, Y. Zhang, Y. Sun, and G. Li, J. Mater. Sci.: Mater. Electron. 26, 9495 (2015). 